\title{
Droxidopa Persistence in Neurogenic Orthostatic Hypotension May Be Affected by Titration Approach
}

\author{
Fahd Amjad' \\ Brett Polenchar ${ }^{2}$ \\ Antonella Favit ${ }^{3}$ \\ 'Department of Neurology, Georgetown \\ University Hospital Pasquerilla \\ Healthcare Center, Washington, DC, \\ USA; ${ }^{2}$ Business Insights, Lundbeck, \\ Deerfield, IL, USA; ${ }^{3}$ Medical Affairs, \\ Lundbeck, Deerfield, IL, USA
}

\begin{abstract}
Droxidopa is approved for the treatment of neurogenic orthostatic hypotension $(\mathrm{nOH})$ symptoms and requires patients to be titrated to individualized effective doses $(100$ $600 \mathrm{mg}$, three times daily) based on symptomatic response. As per the product label, droxidopa should be titrated every 24-48 hours to an optimum maintenance dose (maximum daily dosage $1,800 \mathrm{mg})$. In an examination of patients with $\mathrm{nOH}$ treated in clinical practice settings $(n=4,506)$ using data from the central Northera specialty-pharmacy hub, titration schedules, daily titration dosage (ie, dosage during first dispensation, the assumed titration period), and daily maintenance dosage (dosage during subsequent dispensations) were characterized. It was found that customized titration schedules (ie, different from the product-label recommendation) had been used in $53 \%$ of patients, and these patients had had an average daily titration dosage of $567 \mathrm{mg}$. In contrast, patients who were titrated as per the label schedule (48 hours, 37\%; 24 hours, 10\%) had daily titration dosages of $1,500-1,650 \mathrm{mg}$. A relationship between treatment persistence (measured by number of refills) and maintenance dosage was identified. Average daily maintenance doses in patients who received 2, 3-6, 7-24, and $>25$ dispensations were 938, 969, 1,069, and $1,167 \mathrm{mg}$, respectively $(P<0.0001)$. In summary, our data suggest that more than half the patients treated with droxidopa in clinical practice settings are not titrated using the schedule recommended on the product label (ie, not 24-48 hours), and as a result receive lower daily dosages of droxidopa than those treated using the recommended titration schedules. Lower daily maintenance dosages of droxidopa were associated with shorter treatment persistence (ie, fewer dispensations). Reasons for discontinuation could not be examined in this study, but further investigation of these persistence data is warranted.
\end{abstract}

Keywords: customized titration, real-world evidence, symptomatic relief

\section{Plain-Language Summary}

People with neurodegenerative diseases often experience low blood pressure after standing, a condition called neurogenic orthostatic hypotension (nOH). Symptoms of nOH, such as dizziness and lightheadedness, can increase patients' risk of falls, affect daily activities, and decrease quality of life. Droxidopa is approved for the treatment of nOH symptoms. When treating patients with droxidopa, health-care providers (HCPs) must titrate them to an individualized dosage that provides optimal relief of $\mathrm{nOH}$ symptoms. Droxidopa's prescribing label recommends starting patients with a $100 \mathrm{mg}$ dose three times daily and increasing the dose every 24 48 hours until symptom improvement is achieved or the maximum recommended dosage of $1,800 \mathrm{mg} /$ day is reached. We used prescribing data to examine titration schedules and dosages used by HCPs when treating patients with droxidopa. Over $50 \%$ of patients treated with droxidopa had not been titrated in accordance with the
Correspondence: Fahd Amjad

Department of Neurology, Georgetown University Hospital Pasquerilla Healthcare Center, 7th Floor, 3800 Reservoir Road, NW, Washington, DC, 20007, USA

Tel +I 30I 7748956

Fax +I 30I 7748957

Email fahdvr@hotmail.com 
product label. These patients received lower dosages than patients who had been titrated as per the label. Dosage appeared to affect the time patients continued with treatment: patients who received the highest dosages remained on treatment the longest. We do not know why patients who received lower dosages stopped treatment sooner, but they may not have been receiving an adequate dosage to improve their symptoms. Because they do not experience sufficient symptomatic improvement, they may stop treatment. When treating patients with droxidopa, HCPs should consider if faster titration and higher doses in accordance with the product label would be beneficial.

\section{Introduction}

The disease burden experienced by patients with neurogenic orthostatic hypotension $(\mathrm{nOH})$, a sustained drop in blood pressure (BP) upon standing that is associated with autonomic dysfunction disorders, ${ }^{1,2}$ can be substantial. Such symptoms as dizziness, lightheadedness, and syncope can increase the risk of falls and fall-related injury, negatively affecting the ability to perform daily activities and decreasing quality of life. ${ }^{3-5}$

Droxidopa 100-600 $\mathrm{mg}$ three times daily (ter in die [TID]), a prodrug of the sympathetic neurotransmitter norepinephrine, ${ }^{6}$ is approved for the treatment of symptoms of $\mathrm{nOH}$ (orthostatic dizziness, lightheadedness, or the "feeling that you are about to black out") in patients with primary autonomic failure (Parkinson disease, multiplesystem atrophy, pure autonomic failure), dopamine $\beta$ hydroxylase deficiency, and nondiabetic autonomic neuropathy. ${ }^{7}$ Because symptomatic $\mathrm{nOH}$ is associated with different underlying pathologies and responses to droxidopa can vary among patients, treatment needs to be titrated to an individualized dosage to achieve optimum symptomatic improvement. Labeling instructions state that droxidopa should be titrated to symptomatic response in increments of $100 \mathrm{mg}$ TID every 24-48 hours, starting at $100 \mathrm{mg}$ TID up to a maximum of $600 \mathrm{mg}$ TID (maximum total dosage $1,800 \mathrm{mg} /$ day). ${ }^{7}$ In clinical trials, titration periods $\leq 2$ weeks have been used to identify participants' individually optimized dosage based on tolerability and symptomatic improvement. $^{8-12}$ The mean optimized total daily droxidopa dosages in these clinical trials were 1,170-1,314 mg (ie, 390-438 mg TID), ${ }^{8-11}$ with $82 \%$ of patients receiving $\geq 900 \mathrm{mg} /$ day (ie, $\geq 300 \mathrm{mg}$ TID). ${ }^{12}$

To better understand titration schedules, daily dosages, and treatment persistence associated with real-world use of droxidopa in clinical practice settings, we analyzed specialty pharmacy-dispensing data at the individual patient level. Herein, we describe our findings, compare them with clinical trial data, and discuss important clinical implications of droxidopa-titration patterns and dosage identification in the treatment of patients with $\mathrm{nOH}$.

\section{Methods}

Our analyses used data from patients prescribed droxidopa and enrolled through the central Northera specialty-pharmacy hub (Lash Group [AmerisourceBergen], Fort Mill, SC, USA). Only patients with a paid 30-day first dispensation were included in our analyses, and it was assumed that the first prescription represented titration. Because the titration schedule (ie, increments of dosage increase) cannot be determined from dispensing data, the average daily dose across the 30-day titration period was determined by assuming that the amount of droxidopa prescribed for the first 30 days was related to clinicians' target for maintenance doses. Subsequent dispensations were assumed to occur posttitration and were considered the maintenance dosage (milligrams of drug dispensed/days supplied). Using the number of refills as a surrogate for persistence, the relationship of maintenance dosage with continued treatment (ie, treatment persistence) was examined. Patients were stratified based on the number of refills of droxidopa: patients having received only two (ie, one titration and one maintenance), three to six (approximating $\leq 180$ days of persistence), seven to 24 (approximating $\leq 2$ years of persistence), and those with $\geq 25$. Comparisons between groups were analyzed using one-way ANOVA and Tukey-Kramer post hoc tests, with significance set at $P<0.05$.

\section{Results}

In this study, 4,506 patients were analyzed, and we found that customized schedules (ie, different from the product-labeling recommendation) had been used for 53\%. Patients on a customized titration schedule received an average daily titration dosage of $567 \mathrm{mg}$, and 52\% received a droxidopa dosage $\leq 300 \mathrm{mg} /$ day. Only $13 \%$ of patients on a customized titration schedule reached an average daily droxidopa dosage $>900 \mathrm{mg}$ versus $82 \%$ of the clinical trial population. ${ }^{12}$ In contrast, average daily titration dosages were almost triple (1,500-1,650 mg) for the $47 \%$ of patients titrated according to the label (48-hour titration, 37\%; 24-hour titration, 10\%). Average daily dosages in patients titrated according to the label were more closely aligned with those used by patients in the clinical trials $(1,170-1,314 \mathrm{mg})$ than with those prescribed according to customized titration schedules. These data indicate that $>50 \%$ of patients treated with droxidopa in 
clinical practice settings are titrated according to physician preference (ie, customized titration) and not according to the recommended schedule on the product label or methods used in registration trials. Importantly, customized titration schedules are generally associated with lower daily dosages of droxidopa. However, the reason(s) that customized titration schedules were used by prescribers could not be ascertained from the Northera hub.
The dosage ranges used for the second fill, representing an estimate of the daily maintenance dosage, are shown in Figure 1. For the second fill, average daily dosages $\leq 600 \mathrm{mg}$ were used by 1,796 patients. In our analyses, we also identified a significant relationship between initial daily maintenance dosage of droxidopa (calculated from total milligrams dispensed on second dispensation/days supplied) and treatment persistence (determined by number of refills, $P<0.0001$; Figure 2A). Patients with the

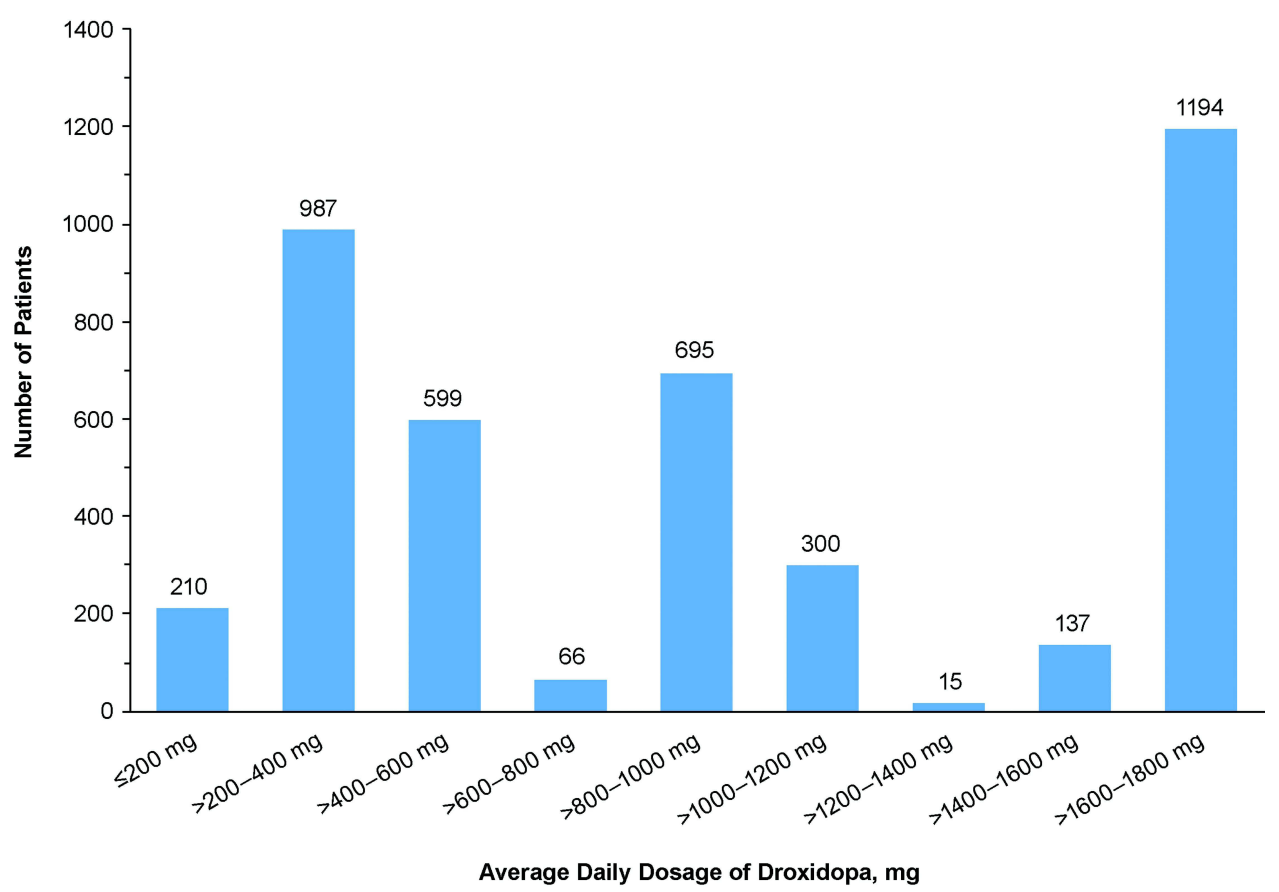

Figure I Average daily dosages of droxidopa at the second fill (ie, maintenance dosage) based on the 30-day supply ordered by the prescriber.

A

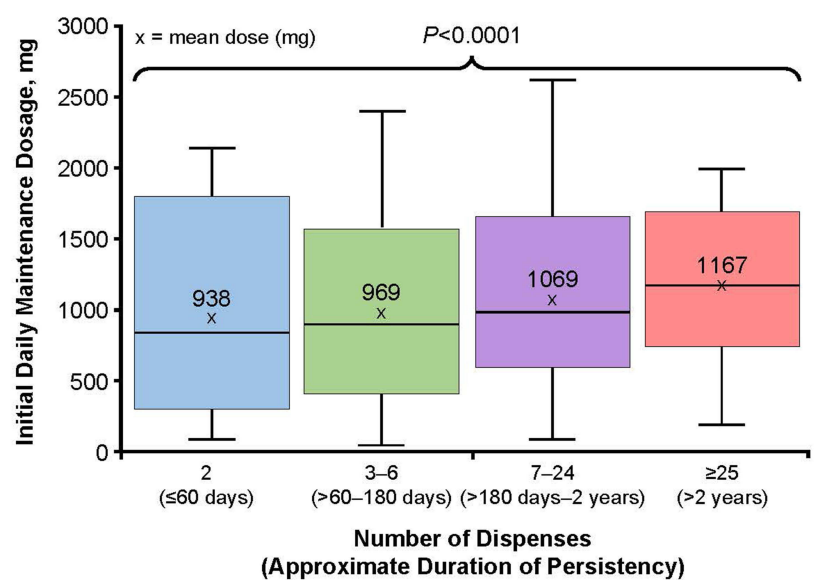

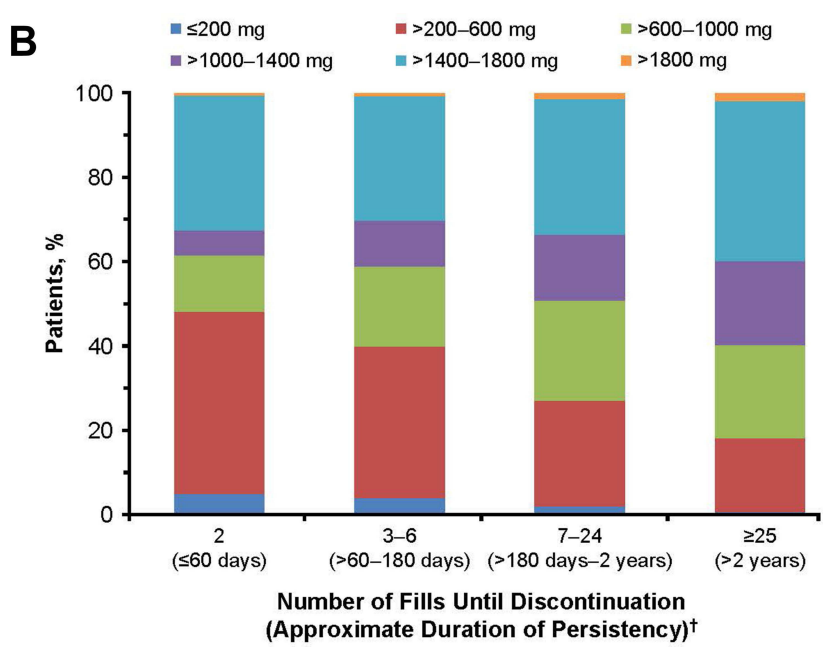

Figure 2 (A) Box plot of initial daily maintenance dosage by number of dispensations. (B) Distribution of average daily maintenance dosage in persistence groupings.**For patients with only two fills (ie, one titration and one maintenance), data represent dose on the second dispensation. All other categories represent average daily dose across all dispensations after the first (ie, titration). ${ }^{\dagger}$ Number of refills received may not align with actual time on therapy, because patients' prescribed daily regimens could not be determined from the data. Discontinuation was defined as no prescription refill within 30 days of the previous fill having run out . 
fewest dispensations (only two fills) began treatment at the lowest average initial daily maintenance dosage (938 mg), and those with the most dispensations $(\geq 25)$ started at the highest average initial daily maintenance dosage (1,$167 \mathrm{mg}$ ). Examination of dosage-level stratification by number of dispensations also supports a relationship between greater persistence and higher average daily dosage (Figure 2B). Notably, this analysis showed that nearly half the patients (48\%) who discontinued after two dispensations had received a dosage $\leq 600 \mathrm{mg} /$ day, whereas most patients $(82 \%)$ who continued to receive droxidopa for $>2$ years received $>600 \mathrm{mg} /$ day. Taken together, our analyses of real-world clinical practice data indicate that many patients are not titrated to clinically relevant doses and that there is a relationship between higher maintenance dosages and greater treatment persistence.

\section{Discussion}

Although droxidopa treatment can be effective at all approved dose levels (100-600 mg TID) and optimal dosage will vary for each patient, treatment for most patients $(>80 \%)$ in the clinical trials was titrated to a maintenance dose $\geq 300 \mathrm{mg}$ TID ( $\geq 900 \mathrm{mg} /$ day). ${ }^{12}$ Although the relationship between daily titration and final maintenance dosage was not established in our analyses, it can be assumed that they are strongly related. Our real-world clinical evidence suggests that customized titration schedules may lead to the identification and use of lower daily maintenance dosages of droxidopa and eventual lack of treatment persistence. This suggests that some patients may prematurely discontinue due to a perception of treatment failure when their dosage may have been suboptimal.

It is important that clinicians recognize potential negative clinical outcomes associated with the identification of suboptimal droxidopa treatment dosages that could result from insufficient titration practices. Slow dosage increases during titration, and possible underdosing of patients is not only undesirable (eg, delaying relief from the burdensome symptoms of nOH) but also could have profound and potentially dangerous consequences (eg, decreasing ability to function in daily activities, increasing risk of falls and injuries). Further, we believe that effects of underdosing may contribute to the association between lower droxidopa dosages and persistence (ie, fewer dispensations) identified in our study. Because patient perception of medication benefit is an important contributor to treatment persistence, ${ }^{13}$ underdosing may cause patients to become frustrated by slow or inadequate symptomatic improvement and then prematurely discontinue treatment because of this perceived lack of benefit. However, because reasons for discontinuation were not available in our data source, we cannot dismiss the possibility that some patients discontinued for reasons other than lack of efficacy (eg, adverse events, out-of-pocket costs, insuranceplan approval, medication burden).

Prescribers should ensure that patients are efficiently titrated to achieve a symptomatic response that is well tolerated, thus allowing the patients to experience relief of $\mathrm{nOH}$ symptoms with the initiation of droxidopa treatment. Careful monitoring of patient progress during droxidopa titration and treatment is essential, and clinicians should aim to identify each patient's individually optimized dosage based on symptomatic and functional improvement promptly by increasing the dose in a manner consistent with the product-label recommendation and clinical trial methods. When titration needs to be stopped because of lack of tolerability (eg, adverse effects, occurrence of supine hypertension), it is important that treatment not be stopped altogether. Rather, maintenance treatment should be initiated at the previous titration dose, assuming it provided reasonable efficacy, safety, and tolerability. Data from a retrospective single-center chart review showed that cognitive and behavioral symptoms emerged in a few patients (six of 101) during dose escalation of droxidopa. However, in most of these patients (four of six), these effects resolved after dose reduction, ${ }^{14}$ which suggests that reducing the dosage of droxidopa to maintain a balance of efficacy, safety, and tolerability is viable.

During droxidopa titration and maintenance treatment, tolerability evaluations should include periodic measurements of supine BP, because any antihypotensive treatment for $\mathrm{nOH}$ can increase the risk of supine hypertension. ${ }^{15} \mathrm{It}$ is recommended that supine BP be measured by the patient at home during initial titration, dose escalation, and based on clinical experience once a week during stable treatment. Patients with supine systolic BP $>180 \mathrm{mmHg}$ or diastolic BP $>110 \mathrm{mmHg}$ (ie, severe supine hypertension) during these home BP checks should be considered for treatment with short-acting antihypertensive agents by their treating physician. Home BP monitoring can be supplemented by in-office BP checks at the physician's discretion. ${ }^{3}$

Although reasons for use of customized titration schedules could not be examined in our study, some prescribers 
may employ these nonrecommended titration regimens as a measure of caution (eg, to mitigate potential tolerability concerns with more rapid titration). Overall clinical trial data on droxidopa suggest that increasing the dose to individual tolerance using the titration schedule on the label should not be a safety concern, nor should it be precluded outright. However, it is important to recognize that patient populations treated in clinical practice may differ from those in clinical trials (eg, comorbidities, underlying cause of $\mathrm{nOH}$ ), which is a reason that droxidopa should be individually titrated.

Our real-world data indicate that droxidopa titration and dosing regimens in clinical practice often differ from the clinical development experience, although we were unable to fully examine the reasons for the observed variations. It is possible that patients treated in real-world clinical practice settings had characteristics (eg, disease severity, demographics, comorbidities) different from patients eligible for inclusion in the clinical trials that affected the outcomes examined (eg, dosage selection, titration schedule, treatment persistence). Physicians should monitor patient progress appropriately during droxidopa treatment to ensure that patients experience optimal symptomatic and functional improvement.

In the randomized clinical trials, droxidopa was generally well tolerated, with adverse events of headache, dizziness, nausea, and hypertension most commonly reported. ${ }^{12}$ Rates of supine hypertension in patients receiving droxidopa in short-term studies (1-8 weeks' exposure) were low $(3.1 \%-7.9 \%){ }^{12}$ Supine hypertension rates in long-term treatment studies (up to 24 months) were $3.8 \%-12.3 \%{ }^{16,17}$

There are some limitations that warrant caution when interpreting real-world clinical data and comparisons with clinical study data. The observational cross-sectional design of this study limits the ability to draw strong conclusions about the relationship between titration dosage/ regimens and treatment persistence. Additionally, the reasons for using customized titration schedules could not be examined in our study. Some prescribers may use these customized titration regimens to mitigate potential tolerability concerns with more rapid titration. Further, patient adherence and compliance could not be determined; therefore, the number of refills a patient had received may not accurately reflect treatment persistence. Finally, the association between titration dosage and relief of $\mathrm{nOH}$ symptoms was not evaluated.

\section{Conclusion}

Our analyses of clinical practice data suggest that prescribers often customize titration of droxidopa using relatively lower dosages than those shown to be effective in clinical development trials. Our key findings that titration dosage is related to the titration method and that treatment persistence is related to maintenance dosage are important and clinically relevant considerations in the treatment of patients with $\mathrm{nOH}$. If patients do not experience symptomatic relief, treatment discontinuation may occur because of a perceived lack of benefit. Because suboptimal dosing may lead to poor clinical outcomes (eg, inadequate relief from nOH symptoms, impaired ability to perform daily activities, risk of falls), prescribers should consider if patients might benefit from higher doses and faster titration when initiating droxidopa treatment.

\section{Abbreviations}

$\mathrm{BP}$, blood pressure; $\mathrm{nOH}$, neurogenic orthostatic hypotension; TID, ter in die (three times daily).

\section{Data Sharing Statement}

The data sets analyzed during the current study are available from the corresponding author on reasonable request.

\section{Research Ethics}

Because these analyses of real-world evidence used aggregated anonymous patient-prescription data, institutional review board/patient consent was not required for the study.

\section{Acknowledgments}

The authors acknowledge the input of and intellectual contributions of L Arthur Hewitt (an employee of Lundbeck at the time of the study), $\mathrm{PhD}$, Steven Kymes, $\mathrm{PhD}$, MHA, and Annika Lindsten, BSc (both current employees of Lundbeck). Medical writing assistance was provided by Lisa Havran, PhD, CMPP, and Patrick Little, $\mathrm{PhD}$, employees of ICON (North Wales, PA).

\section{Author Contributions}

All authors made substantial contributions to conception and design, acquisition of data, or analysis and interpretation of data, took part in drafting the article or revising it critically for important intellectual content, agreed to submit to the current journal, gave final approval to the 
version to be published, and agree to be accountable for all aspects of the work.

\section{Funding}

The data reported were from a study by Lundbeck. Lundbeck provided funding for medical writing and editorial support for the development of the manuscript.

\section{Disclosure}

Fahd S Amjad is a consultant for AbbVie, Acadia, Adamas, Amneal Pharmaceuticals, Kyowa Kirin, Lundbeck, Neurocrine, Teva, Sunovion, and US World Meds. Brett Polenchar is an employee of Lundbeck, and Antonella Favit was an employee of Lundbeck at the time of this study. The authors report no other conflicts of interest in this work.

\section{References}

1. Freeman R, Wieling W, Axelrod FB, et al. Consensus statement on the definition of orthostatic hypotension, neurally mediated syncope and the postural tachycardia syndrome. Clin Auton Res. 2011;21(2):69-72. doi:10.1007/s10286-011-0119-5

2. Freeman R, Abuzinadah AR, Gibbons C, Jones P, Miglis MG, Sinn DI. Orthostatic hypotension: JACC state-of-the-art review. $\mathrm{J} \mathrm{Am}$ Coll Cardiol. 2018;72(11):1294-1309. doi:10.1016/j.jacc.2018.05.079

3. Gibbons CH, Schmidt P, Biaggioni I, et al. The recommendations of a consensus panel for the screening, diagnosis, and treatment of neurogenic orthostatic hypotension and associated supine hypertension. J Neurol. 2017;264(8):1567-1582. doi:10.1007/s00415016-8375-x

4. Claassen DO, Adler CH, Hewitt LA, Gibbons C. Characterization of the symptoms of neurogenic orthostatic hypotension and their impact from a survey of patients and caregivers. BMC Neurol. 2018;18 (1):125. doi:10.1186/s12883-018-1129-x

5. Merola A, Romagnolo A, Rosso M, et al. Autonomic dysfunction in Parkinson's disease: a prospective cohort study. Mov Disord. 2018;33 (3):391-397. doi:10.1002/mds.27268

6. Kaufmann H, Saadia D, Voustianiouk A, et al. Norepinephrine precursor therapy in neurogenic orthostatic hypotension. Circulation. 2003;108(6):724-728. doi:10.1161/01.CIR.0000083721.49847.D7
7. Northera ${ }^{\circledR}$ (droxidopa). Full Prescribing Information. Deerfield, IL: Lundbeck NA Ltd; 2017.

8. Kaufmann H, Freeman R, Biaggioni I, et al. Droxidopa for neurogenic orthostatic hypotension: a randomized, placebo-controlled, Phase 3 trial. Neurology. 2014;83(4):328-335. doi:10.1212/ WNL.0000000000000615

9. Biaggioni I, Freeman R, Mathias CJ, et al. Randomized withdrawal study of patients with symptomatic neurogenic orthostatic hypotension responsive to droxidopa. Hypertension. 2015;65(1):101-107. doi:10.1161/HYPERTENSIONAHA.114.04035

10. Hauser RA, Isaacson S, Lisk JP, Hewitt LA, Rowse G. Droxidopa for the short-term treatment of symptomatic neurogenic orthostatic hypotension in Parkinson's disease (nOH306B). Mov Disord. 2015;30 (5):646-654. doi:10.1002/mds.26086

11. Hauser RA, Hewitt LA, Isaacson S. Droxidopa in patients with neurogenic orthostatic hypotension associated with Parkinson's disease (NOH306A). J Parkinsons Dis. 2014;4(1):57-65. doi:10.3233/ JPD-130259

12. Biaggioni I, Hewitt LA, Rowse GJ, Kaufmann H. Integrated analysis of droxidopa trials for neurogenic orthostatic hypotension. BMC Neurol. 2017;17(1):90. doi:10.1186/s12883-017-0867-5

13. Liu-Seifert H, Adams DH, Ascher-Svanum H, Faries DE, Kinon BJ. Patient perception of medication benefit and early treatment discontinuation in a 1-year study of patients with schizophrenia. Patient Prefer Adherence. 2007;1:9-17.

14. McDonell KE, Shibao CA, Biaggioni I, Hartman A, Robertson D, Claassen DO. Cognitive and behavioral changes in patients treated with droxidopa for neurogenic orthostatic hypotension: a retrospective review. Cogn Behav Neurol. 2019;32(3):179-184. doi:10.1097/WNN.0000000000000198

15. Fanciulli A, Jordan J, Biaggioni I, et al. Consensus statement on the definition of neurogenic supine hypertension in cardiovascular autonomic failure by the American Autonomic Society (AAS) and the European Federation of Autonomic Societies (EFAS): endorsed by the European Academy of Neurology (EAN) and the European Society of Hypertension (ESH). Clin Auton Res. 2018;28 (4):355-362. doi:10.1007/s10286-018-0529-8

16. Isaacson S, Shill HA, Vernino S, Ziemann A, Rowse GJ. Safety and durability of effect with long-term, open-label droxidopa treatment in patients with symptomatic neurogenic orthostatic hypotension (NOH303). J Parkinsons Dis. 2016;6(4):751-759. doi:10.3233/JPD160860

17. Isaacson S, Vernino S, Ziemann A, Rowse GJ, Kalu U, White WB. Long-term safety of droxidopa in patients with symptomatic neurogenic orthostatic hypotension. J Am Soc Hypertens. 2016;10 (10):755-762. doi:10.1016/j.jash.2016.07.010
International Journal of General Medicine

\section{Publish your work in this journal}

The International Journal of General Medicine is an international, peer-reviewed open-access journal that focuses on general and internal medicine, pathogenesis, epidemiology, diagnosis, monitoring and treatment protocols. The journal is characterized by the rapid reporting of reviews, original research and clinical studies across all disease areas. The manuscript management system is completely online and includes a very quick and fair peer-review system, which is all easy to use. Visit http://www.dovepress.com/ testimonials.php to read real quotes from published authors. 